\title{
Common and Dissociable Dysfunction of the Reward System in Bipolar and Unipolar Depression
}

\author{
Theodore D Satterthwaite, ${ }^{*, 1}$ Joseph W Kable ${ }^{2}$, Lillie Vandekar', Natalie Katchmar', Danielle S Bassett ${ }^{3}$, \\ Claudia F Baldassano', Kosha Ruparel', Mark A Elliott', Yvette I Sheline', Ruben C Gur ${ }^{1,4}$, Raquel E Gur ${ }^{1,4}$, \\ Christos Davatzikos ${ }^{4}$, Ellen Leibenluft ${ }^{5}$, Michael E Thase' and Daniel H Wolf' \\ 'Department of Psychiatry, University of Pennsylvania, Philadelphia, PA, USA; ${ }^{2}$ Department of Psychology, University of Pennsylvania, Philadelphia, \\ PA, USA; ${ }^{3}$ Department of Electrical, Systems, and Bioengineering, University of Pennsylvania, Philadelphia, PA, USA; ${ }^{4}$ Department of Radiology, \\ University of Pennsylvania, Philadelphia, PA, USA; ${ }^{5}$ National Institutes of Mental Health Emotion and Development Branch, Bethesda, MD, USA
}

\begin{abstract}
Unipolar and bipolar depressive episodes have a similar clinical presentation that suggests common dysfunction of the brain's reward system. Here, we evaluated the relationship of both dimensional depression severity and diagnostic category to reward system function in both bipolar and unipolar depression. In total, 89 adults were included, including 27 with bipolar depression, 25 with unipolar depression, and 37 healthy comparison subjects. Subjects completed both a monetary reward task and a resting-state acquisition during 3 T BOLD PMRI. Across disorders, depression severity was significantly associated with reduced activation for wins compared with losses in bilateral ventral striatum, anterior cingulate cortex, posterior cingulate cortex, and right anterior insula. Resting-state connectivity within this reward network was also diminished in proportion to depression severity, most notably connectivity strength in the left ventral striatum. In addition, there were categorical differences between patient groups: resting-state connectivity at multiple reward network nodes was higher in bipolar than in unipolar depression. Reduced reward system task activation and resting-state connectivity therefore appear to be a brain phenotype that is dimensionally related to depression severity in both bipolar and unipolar depression. In contrast, categorical differences in reward system resting connectivity between unipolar and bipolar depression may reflect differential risk of mania. Reward system dysfunction thus represents a common brain mechanism with relevance that spans categories of psychiatric diagnosis.

Neuropsychopharmacology (20I5) 40, 2258-2268; doi:I0.1038/npp.20I5.75; published online 8 April 2015
\end{abstract}

\section{INTRODUCTION}

A major depressive episode can occur in the context of both unipolar major depressive disorder (MDD) and bipolar disorder (BP). Although bipolar disorder is distinguished by the presence of mania, the same criteria are used to diagnose depressive episodes in both disorders, and depression is responsible for the majority of overall morbidity and mortality in bipolar disorder (Post, 2005). Despite this, the neurobiology of bipolar depression has been only sparsely explored. The need for further research is underscored by the fact that none of the antidepressant medications approved for treatment of unipolar depression have been approved for treatment of bipolar depression and may carry risks of inducing mania (Ghaemi et al, 2004; Connolly and Thase, 2011). Conversely, the approved pharmacotherapies for bipolar depression remain limited in number, often carry significant risks of metabolic side effects, and are not

* Correspondence: Dr TD Satterthwaite, Department of Psychiatry, Hospital of the University of Pennsylvania, University of Pennsylvania, I Oth Floor, Gates Building, 3400 Spruce Street, Philadelphia, PA 19104, USA. Tel: + I 215662 7119, Fax: + I 2156627903 ,

E-mail: sattertt@upenn.edu

Received 30 October 2014; revised 3 February 2015; accepted 13 February 2015; accepted article preview online 13 March 2015 approved as monotherapy for major depression (Ghaemi et al, 2004; Connolly and Thase, 2011). Similarities of clinical presentation but the presence of differential risk of mania and treatment response suggests that bipolar and unipolar depression have both common and dissociable neurobiological substrates (Treadway and Zald, 2011; Chase et al, 2013). However, relatively little work has directly examined neurobiological phenotypes in both unipolar and bipolar depression (Chase et al, 2013). Here, we identify both common and dissociable neurobiological correlates of bipolar and unipolar depression in reward system activation and connectivity.

Symptoms of depression including anhedonia implicate a hypo-responsive reward system in the pathophysiology of depression (Pizzagalli et al, 2005, 2009; Treadway and Zald, 2011). A wealth of data from both animal studies and human neuroimaging links reward processing to a brain network centered upon the ventral striatum (VS), but also including midbrain, ventromedial prefrontal cortex, posterior cingulate cortex, anterior cingulate cortex, anterior insula, and thalamus (Knutson et al, 2001; Satterthwaite et al, 2007; Kable and Glimcher, 2009). This core reward network was recently confirmed by a comprehensive meta-analysis conducted by Bartra et al (2013). Notably, one subset of regions, including ventromedial prefrontal cortex, posterior cingulate cortex, 
and portions of ventral striatum, shows greater activation to rewards than to punishments, and therefore appears to track the value or valence of rewards. However, another subset of regions shows activation for both rewards and punishments, and therefore appears to track the arousal or salience of rewards; these regions include the anterior cingulate, thalamus, anterior insula, and portions of the ventral striatum. (We refer to the union of these two sets of regions as the 'reward system.') Notably, other work has established that the ventral striatum responds to both reward salience and reward value (Zink et al, 2003, 2004, 2006). Bartra et al (2013) found that value responses are more localized to anterior/ventral VS regions, whereas salience responses tend to be more localized to the posterior/dorsal aspect. Furthermore, resting-state functional connectivity studies have shown that connectivity among reward value, reward salience, and other decisionmaking regions can predict individual differences in rewardrelated decision making, suggesting that connectivity at rest may reflect important properties of reward system function that impact behavior relevant for psychiatric disorders (Li et al, 2013).

Evidence for dysfunction of the brain's reward system in mood disorders is accumulating. In unipolar depression, behavioral studies have demonstrated blunted responsiveness to reward feedback (Pizzagalli et al, 2005, 2008; Steele et al, 2007), and neuroimaging studies have similarly found diminished reward system responses using various task paradigms (Pizzagalli et al, 2009; Smoski et al, 2009; Robinson et al, 2012; Stoy et al, 2012; Dillon et al, 2013). In contrast, several studies in non-depressed subjects with bipolar disorder have found evidence of reward system hyper-responsivity. Elevated reward system responses have been reported in both manic (Abler et al, 2008) and euthymic bipolar patients (Nusslock et al, 2012; Caseras et al, 2013). However, only one study to our knowledge has examined reward-related signaling in bipolar depression: Chase et al (2013) who did not find differences in ventral striatum prediction error responses between controls and subjects with bipolar or unipolar depression. Additionally, an important recent study by Hägele et al (2014) demonstrated that depressive symptoms are associated with a similar blunting of reward responses in a large and diverse sample of subjects including unipolar depression, bipolar disorder, ADHD, alcoholism, and schizophrenia.

While there is ample evidence for abnormalities in the functional connectivity of large-scale brain networks in both major depression (Greicius et al, 2007) and bipolar disorder (Anand et al, 2009), only a few studies have directly compared unipolar and bipolar depression (Liu et al, 2012; Marchand et al, 2013). Abnormalities in cortico-striatal connectivity have been associated with severity of anhedonia in adolescents with major depression (Gabbay et al, 2013), but it is unknown if such abnormalities of connectivity in critical nodes of the reward system are present in bipolar depression, and if severity of depression is related to similar dysfunction of reward circuitry across both bipolar and unipolar depression.

Here, we tested the hypothesis that dimensional dysfunction of the reward system is a critical mechanism underlying the symptoms of both unipolar and bipolar depression using both task-related fMRI and resting-state functional connectivity. We predicted that depression severity would correlate with both diminished activation and resting-state connectivity within the reward system. However, as divergent risk of mania and diverse medication responses suggests categorical differences, we also evaluated group differences between bipolar depression, unipolar depression, and healthy controls. As described below, we found evidence for between-group differences in the mean level of activation and connectivity in the reward system, as well as a common dimensional reduction in activation and connectivity that scaled with depressive symptoms across clinical diagnostic categories.

\section{MATERIALS AND METHODS}

\section{Study Design}

The study included two half-day visits (mean interval between visits: 11.3 days). After complete description of the study to the subjects, written informed consent was obtained; the University of Pennsylvania's Institutional Review Board approved all study procedures. On the first study visit, subjects' diagnosis was assessed for inclusion and exclusion using the Structured Clinical Interview for DSM-IV (First et al, 1996). On the second visit, depression was assessed using the Beck Depression Inventory II (BDI; Beck et al, 1996) and neuroimaging was performed.

\section{Subjects}

Mood disorder subjects were eligible for inclusion if they met criteria for a current depressive episode in the context of either major depressive disorder or bipolar disorder (type I or II). All participants were recruited from outpatient mood disorder clinics. Subjects could not be enrolled if they had a history of substance abuse or dependence (excluding nicotine) in the past 6 months by history or a positive urine drug screen on the day of the study, or any history of pathological gambling screened using items from the South Oaks Gambling Screen. No subjects had received ECT within 6 months of study enrollment. Control subjects were excluded if they met criteria for any Axis I psychiatric disorder over their lifetime.

On initial assessment, all included subjects met criteria for a major depressive episode. However, at time of neuroimaging when the BDI was completed, several subjects $(n=5$ in monetary reward task sample and $n=6$ in resting-state sample) reported only minimal depressive symptoms. While increased range of depressive symptoms enhanced power to detect dimensional associations between imaging phenotypes and depression severity, the results reported herein were qualitatively similar when subjects with minimal depressive symptoms at time of scan were excluded.

Functional imaging data were acquired on 90 adults in total before quality assurance. Of these subjects, 88 had complete data from the monetary reward task. However, 11 subjects were excluded from the final analysis sample of the monetary reward task due to failure to perform the task at a basic level ( $>4$ nonresponses per run or invariant choices in the task; $n=8$ ), excessive in-scanner motion (mean relative displacement $>0.5 \mathrm{~mm} ; n=1$ ), or poor image coverage of the brain $(n=2)$. Thus, following quality assurance, 77 subjects were included in final analysis of the task fMRI data, 
including 23 subjects with bipolar depression (21 bipolar type I), 22 with unipolar depression, and 32 healthy controls. Task-related fMRI data from control participants were included as part of a previous report on amotivation in schizophrenia (Wolf et al, 2014), but was collected over approximately the same time period as that of the mood disorder subjects using identical study procedures.

Resting-state functional connectivity data were available for 87 subjects. Notably, the resting-state scan provided independent data from the reward task acquisition, allowing convergent analyses of reward system function outside task conditions. Of the 87 subjects studied, data from 4 subjects were excluded due to excessive in-scanner motion. Because resting-state functional connectivity data are particularly sensitive to the confounding influences of motion artifact (Satterthwaite et al, 2012), as in our prior work we used a lower threshold of $0.3 \mathrm{~mm}$ mean relative displacement for exclusion (Satterthwaite et al, 2013a, b). Nonetheless, as described below, motion was still included as a covariate in all group level models. The final sample for resting-state data comprised 83 subjects, including 27 subjects with bipolar depression (25 bipolar type I), 23 patients with unipolar major depression, and 33 healthy controls. All mood disorder patients were receiving medication (see Supplementary Table S1). Overall, 89 of the 90 subjects for whom data were acquired were included in either the monetary reward or resting-state analyses. Demographics for this sample are detailed in Table 1.

\section{Image Acquisition and fMRI Paradigm}

All imaging data were acquired on a 3T Siemens TIM TRIO scanner with a 32-channel head coil (see Supplementary Table S2). Sequences acquired included a T1-weighted structural image, a B0 field map for distortion correction, two runs of the monetary reward task, and a resting-state acquisition measuring functional connectivity. Two runs of a reward task using facial affective feedback were also performed; results will be presented elsewhere. The monetary reward task (Wolf et al, 2014) was adapted from Delgado et al (2000). In each trial, subjects viewed the back of a card, and guessed whether the front of the card would be red or black (Figure 1a). Correct guesses gained $\$ 5.00$ and incorrect guesses lost $\$ 4.75$. Subjects were instructed that outcomes depended on their guesses, but in fact outcome order was

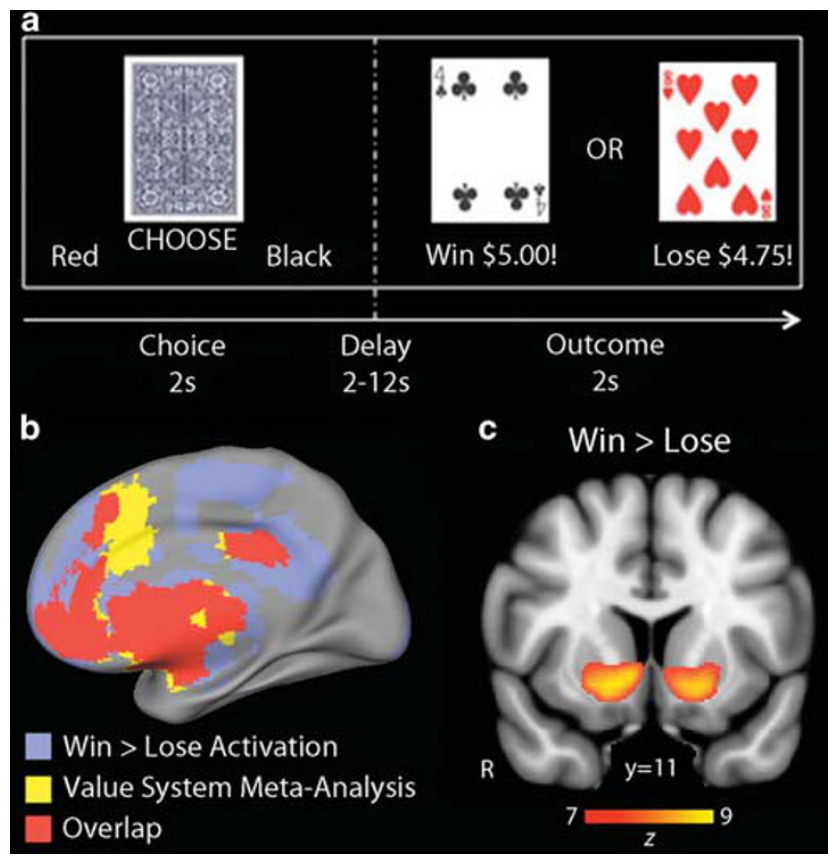

Figure I Task paradigm and activation. (a) Schematic of the fMRI monetary reward paradigm. (b) Win vs loss contrast robustly activates the reward system. The reward system (yellow) as defined by the Bartra et al, meta-analysis encompasses the reward value regions including ventral striatum, ventromedial prefrontal cortex, and posterior cingulate cortex as well as reward salience regions including anterior cingulate, thalamus, and anterior insula. Task activation in the win vs loss contrast (blue) robustly $(z>3.09)$ activates every element of this system with a high degree of overlap (red). (c) Across the whole brain, bilateral ventral striatum was the most strongly activated region.

Table I Sample Demographics

\begin{tabular}{|c|c|c|c|c|}
\hline Variable & Bipolar depression $(n=27)$ & Unipolar depression $(n=25)$ & Healthy controls $(n=37)$ & $P$-value \\
\hline Age (mean, years) & $36.5(11.9)$ & $38.8(12.8)$ & $39.5(11.6)$ & n.s. \\
\hline Gender (\% female) & $63 \%$ & $44 \%$ & $49 \%$ & n.s. \\
\hline Race (\% Caucasian) & $63 \%$ & $48 \%$ & $51 \%$ & n.s. \\
\hline Smoke (\%) & $26 \%$ & $24 \%$ & $30 \%$ & n.s. \\
\hline Beck Depression Inventory (mean total) & $21.75(7.7 I)$ & $25.16(8.63)$ & $1.32(2.15)$ & n.s. ${ }^{a}$ \\
\hline Number of depressive episodes (mean) & 10.5 & 6.8 & NA & n.s. \\
\hline Number of manic episodes (mean) & 6.7 & NA & NA & NA \\
\hline Antipsychotic medication (\%) & $41 \%$ & $8 \%$ & NA & 0.02 \\
\hline Mean dose of antipsychotic medication (CPZ equiv, mg) $)^{b}$ & 342 & 303 & NA & n.s. \\
\hline
\end{tabular}

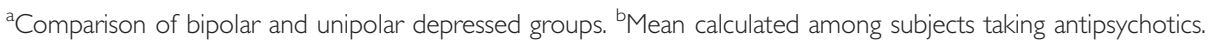


pseudorandomized with an equal number of wins and losses yielding $\$ 6.50$ given to the subject at the end of the study. Each individual trial contained two parts, a guessing phase $(2 \mathrm{~s})$ and an outcome phase ( $2 \mathrm{~s})$, separated by a jittered intra-trial delay $(2-12 \mathrm{~s}$, mean $5 \mathrm{~s})$; inter-trial intervals were jittered the same way. Each card task run comprised $336 \mathrm{~s}$ of analyzed data including 24 trials (12 win and 12 loss). Resting-state imaging was performed on the same subjects (6 min, $12 \mathrm{~s}$ ), during which time subjects were instructed to fixate on a displayed crosshair and remain still.

\section{Structural Image Processing and Functional Image Registration}

To maximize sensitivity to detect effects in small subcortical structures like the ventral striatum, advanced structural image processing and registration procedures were employed. The T1 image was skull stripped using a multi-atlas skull strip procedure (Doshi et al, 2013) followed by multiplicative intrinsic component optimization for bias correction and tissue segmentation (Li et al, 2014). Images were registered to the Montreal Neurological Institute template using a highly-accurate deformable registration with attribute matching and mutual-salience weighting $(\mathrm{Ou}$ et al, 2011). Functional images were distortion-corrected using the B0-map, co-registered to the structural image with boundary-based registration (Greve and Fischl, 2009), and normalized to template space by concatenating all transforms so that only one interpolation was performed.

\section{Image Analysis: Monetary Reward Task}

Monetary reward task image preprocessing and time-series analyses were performed using FEAT in FSL (Jenkinson et al, 2012). As in original reports using this type of paradigm (Delgado et al, 2000), as well as our previous report using this task (Wolf et al, 2014), because of the robust and selective activation of VS to monetary wins compared with monetary losses, our a priori contrast of interest was win > loss outcomes. Notably, while we have previously observed that activation on this contrast is maximal in reward value regions (eg, the vmPFC and anterior-ventral portions of VS), it additionally activates regions involved in saliency processing, including anterior cingulate, anterior insula, and thalamus. This activation may be due to these regions being more sensitive to the salience of rewards than punishments, or to wins being more salient than losses in this task (eg, because the amount won was slightly greater than the amount lost). However, as the task design does not include neutral outcomes, it cannot completely disentangle value and salience effects.

Images were slice-timing corrected, skull-stripped, motion-corrected, high-pass filtered (100 s), spatially smoothed (6 mm FWHM), and grand mean scaled. Subject-level timeseries analysis was carried out using FSL's improved linear model; task regressors (event duration $2 \mathrm{~s}$ ) were convolved with a canonical double-gamma hemodynamic response function. Win and lose trials were modeled as separate regressors during the outcome phase of the task. The guessing phase, outcome phases for non-response trials, rare extraneous button presses, and six motion parameters were all included in the model as nuisance regressors.
Analysis of activation during the card task focused initially on the brain's reward system, followed by exploratory wholebrain analyses. The reward system was defined a priori based on the regions responsive to either positive or negative outcomes in Bartra et al (2013) meta-analysis (Figure 1b). This inclusive definition identifies both areas that primarily respond to reward value and areas that primarily response to reward salience. This approach also allowed us to examine the resting-state connectivity data within a network of regions related to value and salience processing defined in an unbiased manner.

Two group-level analyses were conducted. The first examined group differences using a voxelwise $3 \times 1$ ANOVA. Second, the dimensional effect of depression severity (eg, total BDI) across the two mood groups was evaluated. Both models included sex, age, race, and in-scanner motion as covariates. Notably, group was also accounted for in the analysis of depression severity. Group-by-depression severity interactions were not found in the reward system and thus were not examined further. Significant effects were defined in voxelwise task fMRI data as clusters with a voxel height of $z>2.33$ (uncorrected $P<0.01$ ) and cluster-corrected $P<0.01$, using AFNI AlphaSim. This minimum significant cluster size within the reward system was 90 voxels; for follow-up whole brain exploratory analyses the minimum cluster size was 138 voxels. Images were rendered for display using Mango (http://ric.uthscsa.edu/mango/mango.html).

\section{Image Analysis: Resting-State Functional Connectivity}

We evaluated resting-state functional connectivity using a network analysis of regions implicated in reward value and reward salience processing. Peak coordinates from the Bartra et al (2013) meta-analysis were used to define 11 nodes (Supplementary Table S3) comprising an undirected, weighted network of 53 unique edges. It should be noted that these regions span several classic resting-state networks including the salience, cingulo-opercular, and default mode networks. Briefly, resting-state time-series were distortion corrected, slice time corrected, skull-stripped, realigned, and spatially smoothed at $6 \mathrm{~mm}$ FWHM. To limit the influence of motion artifact, confound regression using a 24 parameter model was performed, including six realignment parameters as well as their squares, temporal derivatives, and square of the temporal derivative (Friston et al, 1996; Satterthwaite et al, 2013a, b; Yan et al, 2013a, 2013b); it should be noted that in this confound regression model white matter, CSF, and global signals are not included. Data were band pass filtered to retain frequencies between 0.01 and $0.08 \mathrm{~Hz}$; the same filter was also applied to confound regressors to prevent frequency mismatch (Hallquist et al, 2013). Registration of processed resting-state time-series to template space was applied as described above. Functional connectivity was calculated for each unique network edge, defined as the $z$-transformed Pearson's correlation between node time-series.

Group-level models evaluated the resting-state connectivity data at two levels of resolution, including (a) overall connectivity strength of each node and (b) connectivity at each network edge. Node strength, defined as the sum of the connectivity strength of each network edge connected to a given node, was calculated using the Brain Connectivity Toolbox (Rubinov and Sporns, 2010). Group-level analyses 
for both nodewise and edgewise data were conducted as for the task data using linear models implemented in R. As for the reward task, two group-level analyses were conducted. The first examined the dimensional effect of depression severity across the two mood groups; the second examined group differences. As for analysis of task data, models included sex, age, race, and in-scanner motion as covariates. Multiple comparisons for both nodewise and edgewise tests were accounted for using the false discovery rate (FDR $Q<0.05$ ). Post hoc pairwise comparisons between groups were computed using the least squares means procedure with a Tukey's correction for multiple comparisons. Network results were rendered for display with custom software written using Mayavi (Ramachandran and Varoquaux, 2011).

\section{Evaluation of Potentially Confounding Variables}

On the basis of prior reports that have documented alterations in reward system responses in association with antipsychotic usage (Abler et al, 2007) or smoking status (Peters et al, 2011), we evaluated the influence of these potentially confounding variables in the present data. Heterogeneity of antidepressants and mood stabilizers used in mood disorder subjects precluded formal evaluation of their impact on observed results (see Limitations, below). Specifically, we re-evaluated significant results on a clusterwise (monetary reward task) or nodewise (resting connectivity) basis with the addition of either smoking status or antipsychotic dose (in chlorpromazine equivalents) as an additional model covariate.

\section{Supplementary Analyses of Additional Symptom Dimensions}

While this study was designed to examine reward system dysfunction in relation to depressive symptomatology in both unipolar and bipolar depression, both sub-domains of depression and other symptom domains may be of interest and were examined as supplementary analyses. Specifically, given prior research relating anhedonia to reward system dysfunction in multiple psychiatric disorders (Juckel et al, 2006; Wolf, 2006; Harvey et al, 2007; Wolf et al, 2008; Pizzagalli et al, 2009; Treadway and Zald, 2011; Gabbay et al, 2013; Hägele et al, 2014) anhedonia was examined using a sub-set of anhedonia-related items from the BDI (Joiner et al, 2003). Furthermore, we similarly examined the relationship with BDI items unrelated to anhedonia. Additionally, the impact of hypomanic spectrum symptoms was assessed in bipolar subjects using the total mania score of the Bipolar Inventory of Symptoms Scale (BISS; Bowden et al, 2007). Models investigating win $v$ s loss activation in the monetary reward task and nodewise connectivity strength in the resting-state data were constructed identically to those used for examination of the BDI as described above.

\section{RESULTS}

\section{Monetary Reward Task Recruits the Reward System}

The win $>$ loss contrast robustly recruited regions known to be involved in reward value and reward salience processing, including ventral striatum, ventromedial prefrontal cortex, thalamus, anterior cingulate, posterior cingulate, and anterior insula. Areas of activation in this contrast overlapped highly with regions identified by the Bartra et al meta-analysis (Figure 1b). While this task cannot fully disentangle value and salience effects, maximal activation in the contrast of interest was observed in regions known to respond more to rewards than to punishments, including the bilateral ventral striatum (Figure 1c) and the ventromedial prefrontal cortex.

\section{Depression Severity Is Related to Blunted Recruitment of Reward System}

Depression severity (total BDI), regardless of clinical diagnosis, was dimensionally associated with diminished activation of the reward system in the win $>$ loss contrast. Across both bipolar and unipolar depressed patients, higher levels of depression were correlated with diminished activation of key hubs of the reward system, including bilateral ventral striatum and posterior cingulate, as well as reward salience regions such as the anterior insula and anterior cingulate (see Figure 2 and Table 2A). When these clusters were examined within each group, similar effects were found in both bipolar and unipolar depression (Supplementary Table S4). Notably, all regions were located within the a priori mask, but effects were also robust enough to survive whole-brain correction for multiple comparisons. Additionally, results remained significant when these clusters were re-evaluated with smoking status or antipsychotic dose included as a model covariate.

\section{Depression Severity Is Associated with Lower Reward Network Resting-State Connectivity}

Examination of connectivity revealed convergent results: dimensional depression severity correlated with diminished

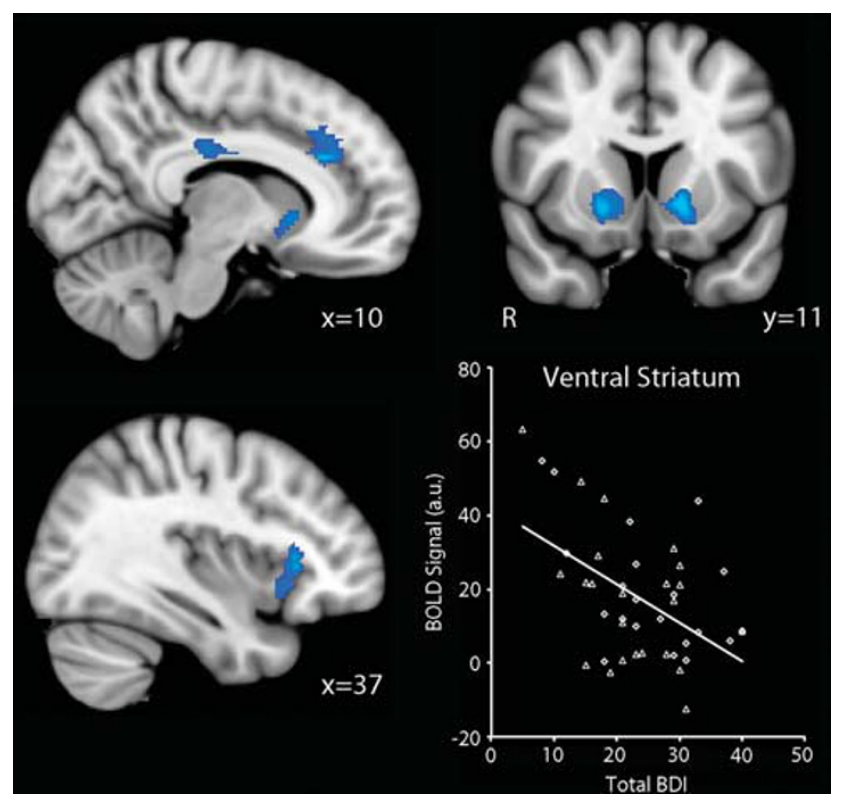

Figure 2 Depression severity is associated with diminished reward system activation. Activation in the win > loss contrast is dimensionally reduced in association with depression severity across both unipolar and bipolar depressed groups. Reward system regions showing a significant $(z>2.3$, whole brain corrected $P<0.01)$ reduction in activation included bilateral ventral striatum, anterior cingulate cortex, posterior cingulate cortex, and right anterior insula. 
Table 2 Neuroimaging Results

\begin{tabular}{|c|c|c|c|}
\hline \multicolumn{4}{|c|}{ A. Association Between Depression Severity (BDI) and Activation on Win $>$ Loss Contrast } \\
\hline Region & MNI coordinates & Cluster size (vox) & $\operatorname{Max}(Z)$ \\
\hline Posterior cingulate & $-10,-32,32$ & 444 & 3.43 \\
\hline Left ventral striatum & $-14,10,-4$ & 352 & 3.44 \\
\hline Right ventral striatum & $18,14,-2$ & 297 & 3.61 \\
\hline Anterior cingulate & $10,30,26$ & 294 & 3.46 \\
\hline \multicolumn{4}{|c|}{ B. Association Between Depression Severity (BDI) and Overall Connectivity Strength of Value Network Nodes } \\
\hline Node & t-Value & Corrected $P$-value & \\
\hline Left ventral striatum & 4.8 & 0.0002 & \\
\hline \multicolumn{4}{|c|}{ C. Association Between Depression Severity (BDI) and Connectivity of Value Network Edges } \\
\hline Right ventral striatum & Left thalamus & 2.3 & 0.03 \\
\hline Right ventral striatum & Right thalamus & 3.8 & 0.008 \\
\hline VmPFC & VTA & 4.1 & 0.005 \\
\hline \multicolumn{4}{|c|}{ D. Group Differences in Overall Connectivity Strength of Value Network Nodes } \\
\hline Node & F-value & Corrected P-value & Effect \\
\hline Left ventral striatum & 4.6 & 0.03 & Control > MDD \\
\hline Left anterior insula & 4.9 & 0.03 & Control > MDD \\
\hline VTA & 6.3 & 0.01 & Control > MDD \\
\hline Anterior cingulate & 6.7 & 0.01 & Control \& BP > MDD \\
\hline
\end{tabular}

resting-state functional connectivity across both unipolar and bipolar depression. Evidence for impaired resting-state functional connectivity was present on both nodal (Table 2B) and edgewise scales of analysis (Table 2C). In particular, nodal connectivity strength of the left ventral striatum exhibited a robust negative relationship with depression severity (partial $r=-0.59$, uncorrected $P=1.8 \times 10^{-5}$; FDR-corrected $P=0.0002$; see Table $2 \mathrm{~B}$ and Figure $3 \mathrm{a}$ and b). This effect was similar in both unipolar (partial $r=-0.65$ ) and bipolar depressed groups (partial $r=-0.58$ ). No dimensional effects survived FDR correction at other network nodes. Results remained significant when smoking status or antipsychotic dose was modeled as a covariate.

Analysis of the dimensional effect of depression on connectivity at individual network edges (Table 2C) also revealed diminished connectivity with greater depression severity, including connectivity between the ventral striatum and the thalamus, as well between as the ventral tegmental area and the ventromedial prefrontal cortex. No network nodes or edges demonstrated increased connectivity with depression severity.

\section{Categorical Differences Between Bipolar and Unipolar Depression}

The dimensional results establish that depression severity in both unipolar and bipolar depression is associated with diminished task activation and reduced functional connectivity at rest within the reward system. We next tested for group differences between these diagnostic groups as well as healthy controls. No clusters survived correction for multiple comparisons in the between-group ANOVA of the win $>$ loss contrast. However, based on prior reports of differences between groups, we conducted exploratory pairwise comparisons which revealed that bipolar subjects had greater activation than unipolar patients in the left ventral striatum, with a sub-threshold effect present in the right ventral striatum as well (Supplementary Figure S1); comparison subjects did not differ significantly from either patient group. No other exploratory pairwise group comparison yielded significant results.

Analysis of connectivity data revealed qualitatively similar but more robust categorical group differences. Nodewise analysis of the resting-state connectivity data revealed that in ventral striatum, anterior insula, VTA, and thalamus, patients with unipolar depression showed reduced nodal connectivity strength relative to both bipolar subjects and healthy comparison subjects (see Table $2 \mathrm{D}$ and Figure $3 \mathrm{c}$ and $\mathrm{d}$ ). These results remained significant when smoking or antipsychotic dose was modeled as covariates. Healthy comparison subjects and those with bipolar depression did not differ in nodal connectivity. No categorical differences between groups survived FDR correction when individual network edges were analyzed. 

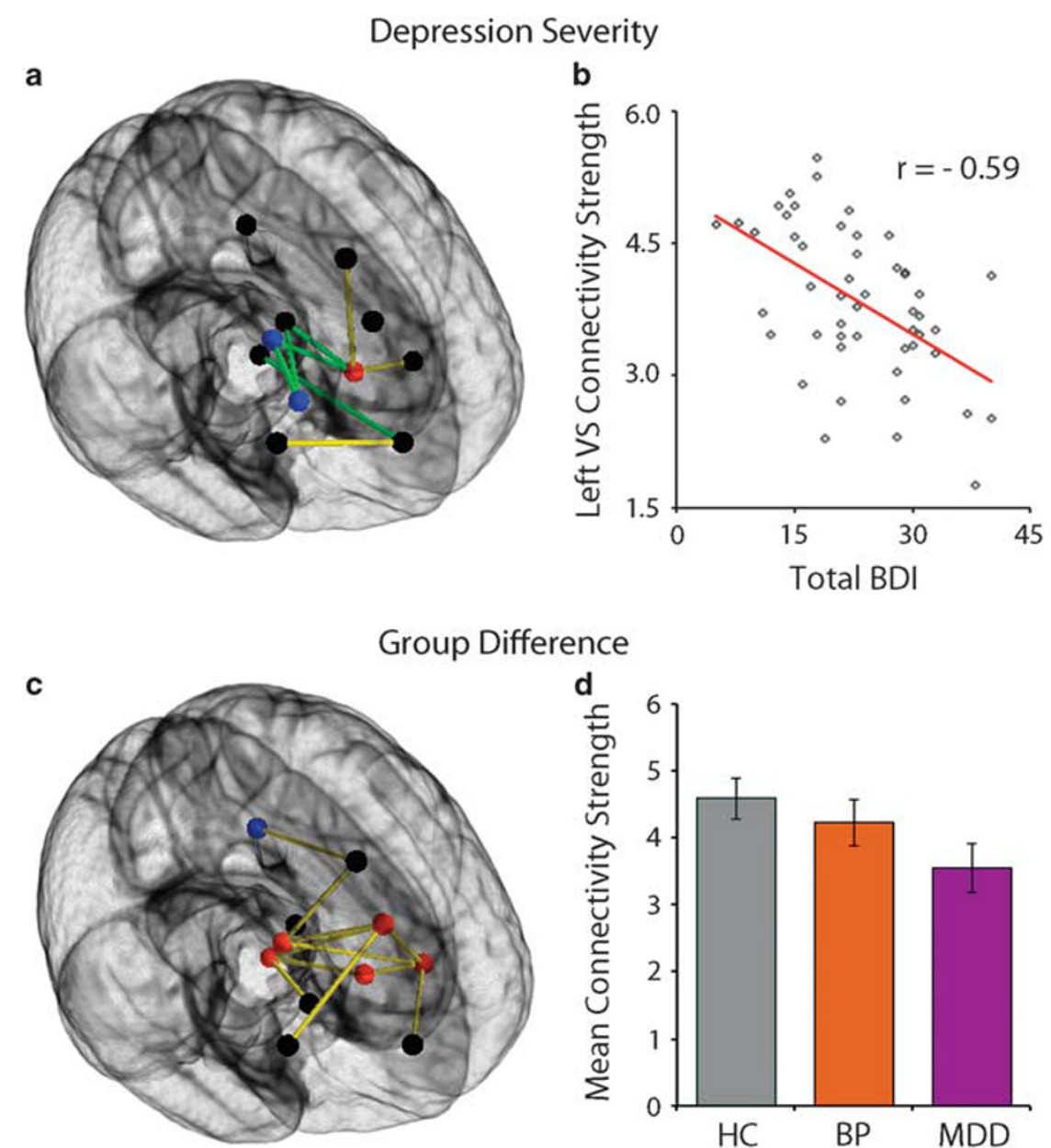

Figure 3 Reward network resting-state connectivity reveals common dimensional effects of depression severity across groups as well as between-group differences. (a) Greater depression severity in both unipolar and bipolar depression was associated with diminished connectivity within the reward network. The most notable association with depression severity was seen at the left ventral striatum, where total reward network connectivity strength was robustly reduced in proportion with depression severity (b; partial $r=-0.59$ ). (c) In addition to dimensional across-group effects of depression severity, between-group differences in reward network connectivity were also observed. In four nodes within the reward system, unipolar depression subjects (MDD) demonstrated diminished connectivity within the reward network compared with healthy controls and bipolar depression subjects (BP). (d) Mean nodal connectivity strength across the four nodes (red) where a group difference was found. Error bars represent 95\% confidence intervals. In (a and c), nodes are colored according to strength of association (red: FDR $Q<0.05$; blue: uncorrected $P<0.05$; black: NS). Edges with a significant FDR-corrected effect are colored green; yellow indicates uncorrected $P<0.05$.

\section{Supplementary Analyses of Additional Symptom Dimensions}

As a final step, we performed supplementary analyses examining anhedonic (and non-anhedonic) symptoms across both groups as well as hypomanic-spectrum symptoms in the bipolar group. Notably, both anhedonic and nonanhedonic depressive symptoms as measured by the BDI were significantly related to reduced VS activation on the win vs loss contrast. However, this relationship was of a similar magnitude to that seen with overall depression severity (total BDI). A trend toward diminished VS connectivity strength was similarly seen in association with anhedonic symptoms, and a significant relationship was seen with non-anhedonic symptoms, but the relationship was less robust than that seen with total depression severity. Finally, no significant relationships between hypomanic symptoms in the bipolar group and task activation or resting-state connectivity in bipolar subjects were observed.

\section{DISCUSSION}

Our results demonstrate that symptoms of depression are related to reduced activation and connectivity of the brain's reward system in both unipolar and bipolar depression. In addition to this common dimensional effect, we found group differences in average levels of reward system connectivity, with greater connectivity in bipolar than in unipolar depression. These results provide novel evidence for common and dissociable neural correlates of depression across mood disorders.

Our results agree with prior work demonstrating reduced brain reward responses in unipolar major depression (Pizzagalli et al, 2009; Smoski et al, 2009; Robinson et al, 
2012; Stoy et al, 2012; Dillon et al, 2013). However, prior studies have typically focused on categorical differences between a given diagnosis and healthy comparators, and have not related reward hypo-responsivity to depression severity across mood disorder diagnostic categories. Our dimensional approach and findings are consistent with the overarching aims of the NIMH Research Domain Criteria (Insel et al, 2010), and demonstrate that blunted reward system responsiveness scales with symptom severity in both bipolar and unipolar depression. Using the same task, we recently found that ventral striatum hypofunction selectively related to the severity of behavioral amotivation in schizophrenia (Wolf et al, 2014). Thus, reward system pathology may contribute to clinical reward dysfunction across multiple psychiatric disorders. Further confirmation comes from an important recent study by Hägele et al (2014), which demonstrated that depressive symptoms are associated with a similar blunting of reward responses in a large sample of patients with diverse diagnoses.

In comparison with imaging studies examining reward system function in unipolar major depression, there is a relative dearth of prior work examining reward system function in bipolar depression. To our knowledge, the only published work was the recent study by Chase et al, who compared reward prediction errors in bipolar and unipolar depression. This study did not identify reductions in ventral striatum activation in either group. While there are multiple differences with the current study, including the paradigm used and the analytic approach, the dimensional approach of our study may have provided greater sensitivity; indeed, for the task activation data, dimensional effects were more robust than group differences.

Results from resting-state connectivity build upon several small prior studies that have examined both unipolar and bipolar depression and have produced somewhat heterogeneous results. Anand et al (2009) reported reduced anterior cingulate connectivity in a mixed sample of unipolar depressed, manic bipolar, and bipolar depressed patients. In a study of bipolar and unipolar depressed patients, Marchand et al (2013) found differences in posterior cingulate connectivity profiles. Most recently, and consistent in part with the current results, Liu et al (2012) reported that insula amplitude of low-frequency fluctuations (ALFF) was higher in bipolar than in unipolar depression, and that lower ALFF was associated with depression severity in both disorders.

The network analysis we conducted focused on the reward system, and revealed that connectivity was reduced in proportion to depression severity across both unipolar and bipolar depression. In particular, the single node that survived correction was the left ventral striatum, which demonstrated a robust negative relationship between depression severity and connectivity strength within the reward network. The concordance with results from task-related fMRI suggests that common dimensional abnormalities of the reward system are present not just during active reward processing but at rest as well, potentially mediating persistent and endogenous reductions in mood.

In addition to this evidence of common across-group effects of depression severity, there were between-group differences in connectivity as well. Results suggest that although common dimensional effects of depression severity on the reward system occur across disorders, these effects are superimposed upon differences in reward system connectivity, where patients with unipolar depression showed diminished connectivity compared with controls and bipolar depression subjects.

Although less robust, a similar effect of diagnosis was also seen in the monetary reward task, where exploratory pairwise comparisons demonstrated higher ventral striatum activation in bipolar depression than in unipolar depression. However, this effect was not significant in the voxelwise ANOVA, and should thus be interpreted with caution. The lack of categorical group differences between either patient group and controls on the monetary reward task was somewhat unexpected, especially for the unipolar depression group, where multiple prior studies have shown blunting of reward system responses (Pizzagalli et al, 2009; Smoski et al, 2009; Robinson et al, 2012; Stoy et al, 2012; Dillon et al, 2013). This may be due to the range of depression severity present in unipolar patients included in the current study; given the dimensional effects we observed, we would expect categorical group effects to be more robust in patient groups with more severe symptoms.

Notably, bipolar depressed patients did not significantly differ from healthy controls on either reward system activation or connectivity. However, prior research suggests that bipolar patients studied during mania or euthymic states may have enhanced reward responses (Abler et al, 2008; Nusslock et al, 2012; Caseras et al, 2013). Although speculative, it is possible that patients with bipolar disorder may have tonically elevated reward system activation and connectivity, which is dimensionally blunted in depressive episodes. The co-occurrence in bipolar disorder of elevated average reward system function and depression-dependent hypofunction in this circuit could potentially reflect antagonistic or compensatory processes that contribute to the cyclicity of mood abnormalities. Such an interpretation is suggested in part by data showing higher levels of behavioral activation (using the BIS/BAS scale) in bipolar than in unipolar patients who were in a major depressive episode (Quilty et al, 2014).

Several limitations should be noted. First, while the results link reward system hypofunction and depression severity, these associations do not establish causation. Future studies employing animal models and treatment interventions can test this link mechanistically. Second, all mood disorder patients in this study were medicated, and while we did not observe any effects of antipsychotic dosage on results, we cannot completely exclude a potential influence of betweengroup differences in medications on observed results. Heterogeneity of the antidepressants and mood stabilizers used precluded formal evaluation of their impact on observed results. However, it seems quite unlikely that the heterogeneous medications within each group would produce the common dimensional effects across groups. Past work has also suggested that medication effects in fMRI studies of mood disorders tend to normalize brain responses (Hafeman et al, 2012), and such effects would not explain the current pattern of results. Third, although the differences we observe between dimensional and categorical analyses suggest distinct and potentially inter-related state and trait abnormalities in reward system function, our cross-sectional sample limits interpretation. Longitudinal studies spanning 
mood state transitions will be required to rigorously disentangle state $v s$ trait effects of reward system dysfunction in mood disorders. Fourth, while the win $v s$ loss contrast of this study most strongly activates regions linked primarily to reward value processing, it also activates regions implicated in reward salience processing. Therefore, although we have primarily interpreted our results in context of literature regarding reward-related dysfunction in mood disorders, future work should attempt to specifically parse deficits in reward value and reward salience systems (Zink et al, 2003, 2004, 2006), which may be distinct in clinical populations.

Finally, recent work in depression (Pizzagalli et al, 2005, 2009; Gabbay et al, 2013), and our own work in schizophrenia (Wolf et al, 2014) suggests the merit of investigating more specific components of psychopathology such as anhedonia (Treadway and Zald, 2011), amotivation, or irritability (Stringaris et al, 2009) rather than overall depression severity. While in this study we did not find a specific relationship between reward system dysfunction and anhedonia-related items on the BDI, more detailed assessments might be more sensitive; the truncated range of anhedonia items from the BDI used here may have limited detection power. Given the prevalence of anhedonic symptoms across both mood and psychotic disorders (Wolf, 2006; Treadway and Zald, 2011; Hägele et al, 2014), such work could be conducted on a dimensional basis across a wider spectrum of disorders as well as in developing populations (Casey et al, 2014). Indeed, recent work by Hägele et al (2014) showed similar dimensional rewardsystem dysfunction in relation to depressive symptoms across multiple disorders including schizophrenia, alcoholism, $\mathrm{ADHD}$, bipolar disorder, and MDD, emphasizing the relevance of such research beyond mood disorders alone.

These limitations notwithstanding, our results suggest that reward system dysfunction may give rise to symptoms of depression in both bipolar depression and unipolar depression. However, these common dimensional changes may occur relative to a different baseline in the two disorders, as reflected by the observed group differences in connectivity. Further efforts to disentangle shared and divergent mechanisms of depression could yield critical advances in our ability to distinguish bipolar and unipolar depression, without relying on a history of manic symptoms. As this history may be difficult to obtain, and since bipolar disorder may present first with a depressive episode, this diagnostic advance would facilitate appropriate treatment of bipolar disorder especially in the early stages. In the future, reward system activation and connectivity have the potential to be useful imaging biomarkers in the context of drug discovery and clinical trials for treatment of mood disorders.

\section{FUNDING AND DISCLOSURE}

The authors declare no conflict of interest.

\section{ACKNOWLEDGMENTS}

Thanks to Monica Calkins for assistance with clinical assessment training, and to Chad Jackson and Larry Macy for systems support. Many thanks to Alex Smith and Ragini Verma for providing network visualization software. Support provided by the Brain and Behavior Foundation through the Marc Rapport Family Investigator Grant and the Sidney $\mathrm{R}$ Baer, Jr. Foundation, the Penn Medicine Neuroscience Center, the American Psychiatric Institute for Research and Education, National Institute of Mental Health Grants K23MH098130, K23MH085096, T32 MH019112, and R01MH101111. Dr Sheline reports a past relationship with Neosynch. Dr Thase reports no conflicts directly pertaining to this research. Over the past 36 months, he reports the following relationships: Alkermes, AstraZeneca, BristolMyers Squibb, Cerecor, Eli Lilly, Dey Pharma, Forest Laboratories, Gerson Lehman Group, Guidepoint Global, H. Lundbeck A/S, MedAvante, Merck, Neuronetics, Otsuka, Ortho-McNeil, Pamlab, Pfizer, PGx, Shire, Sunovion, Supernus, Takeda, and Transcept Pharmaceuticals.

\section{REFERENCES}

Abler B, Erk S, Walter H (2007). Human reward system activation is modulated by a single dose of olanzapine in healthy subjects in an event-related, double-blind, placebo-controlled fMRI study. Psychopharmacology (Berl) 191: 823-833.

Abler B, Greenhouse I, Ongur D, Walter H, Heckers S (2008). Abnormal reward system activation in mania. Neuropsychopharmacology 33: 2217-2227.

Anand A, Li Y, Wang Y, Lowe MJ, Dzemidzic M (2009). Resting state corticolimbic connectivity abnormalities in unmedicated bipolar disorder and unipolar depression. Psychiatry Res 171: 189-198.

Bartra O, McGuire JT, Kable JW (2013). The valuation system: a coordinate-based meta-analysis of BOLD fMRI experiments examining neural correlates of subjective value. Neuroimage 76: 412-427.

Beck AT, Steer RA, Ball R, Ranieri W (1996). Comparison of Beck Depression Inventories -IA and -II in psychiatric outpatients. J Pers Assess 67: 588-597.

Bowden CL, Singh V, Thompson P, Gonzalez JM, Katz MM, Dahl M et al (2007). Development of the bipolar inventory of symptoms scale. Acta Psychiatr Scand 116: 189-194.

Caseras X, Lawrence NS, Murphy K, Wise RG, Phillips ML (2013). Ventral striatum activity in response to reward: differences between bipolar I and II disorders. Am J Psychiatry 170: 533-541.

Casey BJ, Oliveri ME, Insel $\mathrm{T}$ (2014). A neurodevelopmental perspective on the Research Domain Criteria (RDoC) Framework. Biol Psychiatry 76: 350-353.

Chase HW, Nusslock R, Almeida JRC, Forbes EE, LaBarbara EJ, Phillips ML (2013). Dissociable patterns of abnormal frontal cortical activation during anticipation of an uncertain reward or loss in bipolar versus major depression. Bipolar Disord 15: 839-854.

Connolly KR, Thase ME (2011). The clinical management of bipolar disorder: a review of evidence-based guidelines. Prim Care Companion CNS Disord 13: pii: PCC.10r01097.

Delgado MR, Nystrom LE, Fissell C, Noll DC, Fiez JA (2000). Tracking the hemodynamic responses to reward and punishment in the striatum. J Neurophysiol 84: 3072-3077.

Dillon DG, Dobbins IG, Pizzagalli DA (2013). Weak reward source memory in depression reflects blunted activation of VTA/SN and parahippocampus. Soc Cogn Affect Neurosci 9: 1576-1583.

Doshi J, Erus G, Ou Y, Gaonkar B, Davatzikos C (2013). Multi-atlas skull-stripping. Acad Radiol 20: 1566-1576.

First MB, Spitzer RL, Gibbon M, Williams JBW (1996). Structured Clinical Interview for DSM-IV Axis I Disorders, Patient Edition (SCID-P), Version 2. New York State Psychiatric Institute: Biometrics Research: New York. 
Friston KJ, Williams S, Howard R, Frackowiak RS, Turner R (1996). Movement-related effects in fMRI time-series. Magn Reson Med 35: 346-355.

Gabbay V, Ely BA, Li Q, Bangaru SD, Panzer AM, Alonso CM et al (2013). Striatum-based circuitry of adolescent depression and anhedonia. J Am Acad Child Adolesc Psychiatry 52: 628-641, e13.

Ghaemi SN, Rosenquist KJ, Ko JY, Baldassano CF, Kontos NJ, Baldessarini RJ (2004). Antidepressant treatment in bipolar versus unipolar depression. Am J Psychiatry 161: 163-165.

Greicius MD, Flores BH, Menon V, Glover GH, Solvason HB, Kenna $\mathrm{H}$ et al (2007). Resting-state functional connectivity in major depression: abnormally increased contributions from subgenual cingulate cortex and thalamus. Biol Psychiatry 62: 429-437.

Greve DN, Fischl B (2009). Accurate and robust brain image alignment using boundary-based registration. Neuroimage 48: 63-72.

Hafeman DM, Chang KD, Garrett AS, Sanders EM, Phillips ML (2012). Effects of medication on neuroimaging findings in bipolar disorder: an updated review. Bipolar Disord 14: 375-410.

Hallquist MN, Hwang K, Luna B (2013). The nuisance of nuisance regression: spectral misspecification in a common approach to resting-state $\mathrm{fMRI}$ preprocessing reintroduces noise and obscures functional connectivity. Neuroimage 82C: 208-225.

Harvey P-O, Pruessner J, Czechowska Y, Lepage M (2007). Individual differences in trait anhedonia: a structural and functional magnetic resonance imaging study in non-clinical subjects. Mol Psychiatry 12: 703, 767-775.

Hägele C, Schlagenhauf F, Rapp M, Sterzer P, Beck A, Bermpohl F et al (2014). Dimensional psychiatry: reward dysfunction and depressive mood across psychiatric disorders. Psychopharmacology (Berl) 232: 331-341.

Insel T, Cuthbert B, Garvey M, Heinssen R, Pine DS, Quinn K et al (2010). Research domain criteria (RDoC): toward a new classification framework for research on mental disorders. Am J Psychiatry 167: 748-751.

Jenkinson M, Beckmann CF, Behrens TEJ, Woolrich MW, Smith SM (2012). FSL. Neuroimage 62: 782-790.

Joiner TE, Brown JS, Metalsky GI (2003). A test of the tripartite model's prediction of anhedonia's specificity to depression: patients with major depression versus patients with schizophrenia. Psychiatry Res 119: 243-250.

Juckel G, Schlagenhauf F, Koslowski M, Wustenberg T, Villringer A, Knutson B et al (2006). Dysfunction of ventral striatal reward prediction in schizophrenia. Neuroimage 29: 409-416.

Kable JW, Glimcher PW (2009). The neurobiology of decision: consensus and controversy. Neuron 63: 733-745.

Knutson B, Adams CM, Fong GW, Hommer D (2001). Anticipation of increasing monetary reward selectively recruits nucleus accumbens. J Neurosci 21: RC159-RC159.

Li C, Gore JC, Davatzikos C (2014). Multiplicative Intrinsic Component Optimization (MICO) for MRI bias field estimation and tissue segmentation. Magn Reson Imaging 32: 913-923.

Li N, Ma N, Liu Y, He X-S, Sun D-L, Fu X-M et al (2013). Restingstate functional connectivity predicts impulsivity in economic decision-making. J Neurosci 33: 4886-4895.

Liu C-H, Ma X, Wu X, Li F, Zhang Y, Zhou F-C et al (2012). Resting-state abnormal baseline brain activity in unipolar and bipolar depression. Neurosci Lett 516: 202-206.

Marchand WR, Lee JN, Johnson S, Gale P, Thatcher J (2013). Differences in functional connectivity in major depression versus bipolar II depression. J Affect Disord 150: 527-532.

Nusslock R, Almeida JR, Forbes EE, Versace A, Frank E, Labarbara et al (2012). Waiting to win: elevated striatal and orbitofrontal cortical activity during reward anticipation in euthymic bipolar disorder adults. Bipolar Disord 14: 249-260.

Ou Y, Sotiras A, Paragios N, Davatzikos C (2011). DRAMMS: Deformable registration via attribute matching and mutualsaliency weighting. Med Image Anal 15: 622-639.
Peters J, Bromberg U, Schneider S, Brassen S, Menz M, Banaschewski $\mathrm{T}$ et al (2011). Lower ventral striatal activation during reward anticipation in adolescent smokers. $A m \mathrm{~J}$ Psychiatry 168: 540-549.

Pizzagalli DA, Holmes AJ, Dillon DG, Goetz EL, Birk JL, Bogdan R et al (2009). Reduced caudate and nucleus accumbens response to rewards in unmedicated individuals with major depressive disorder. Am J Psychiatry 166: 702-710.

Pizzagalli DA, Iosifescu D, Hallett LA, Ratner KG, Fava M (2008). Reduced hedonic capacity in major depressive disorder: evidence from a probabilistic reward task. J Psychiatr Res 43: 76-87.

Pizzagalli DA, Jahn AL, O'Shea JP (2005). Toward an objective characterization of an anhedonic phenotype: a signal-detection approach. Biol Psychiatry 57: 319-327.

Post RM (2005). The impact of bipolar depression. J Clin Psychiatry 66: 5-10.

Quilty LC, Mackew L, Bagby RM (2014). Distinct profiles of behavioral inhibition and activation system sensitivity in unipolar vs bipolar mood disorders. Psychiatry Res 219: 228-231.

Ramachandran P, Varoquaux G (2011). Mayavi: 3D visualization of scientific data. Comput Sci Eng 13: 40-51.

Robinson OJ, Cools R, Carlisi CO, Sahakian BJ, Drevets WC (2012). Ventral striatum response during reward and punishment reversal learning in unmedicated major depressive disorder. $\mathrm{Am}$ J Psychiatry 169: 152-159.

Rubinov M, Sporns O (2010). Complex network measures of brain connectivity: uses and interpretations. Neuroimage 52: 1059-1069.

Satterthwaite TD, Elliott MA, Gerraty RT, Ruparel K, Loughead J, Calkins ME et al (2013a). An improved framework for confound regression and filtering for control of motion artifact in the preprocessing of resting-state functional connectivity data. Neuroimage 64: 240-256.

Satterthwaite TD, Green L, Myerson J, Parker J, Ramaratnam M, Buckner RL (2007). Dissociable but inter-related systems of cognitive control and reward during decision making: evidence from pupillometry and event-related fMRI. Neuroimage 37: 1017-1031.

Satterthwaite TD, Wolf DH, Loughead J, Ruparel K, Elliott MA, Hakonarson $\mathrm{H}$ et al (2012). Impact of in-scanner head motion on multiple measures of functional connectivity: Relevance for studies of neurodevelopment in youth. Neuroimage 60: 623-632. Satterthwaite TD, Wolf DH, Ruparel K, Erus G, Elliott MA, Eickhoff SB et al (2013b). Heterogeneous impact of motion on fundamental patterns of developmental changes in functional connectivity during youth. Neuroimage 83: 45-57.

Smoski MJ, Felder J, Bizzell J, Green SR, Ernst M, Lynch TR et al (2009). fMRI of alterations in reward selection, anticipation, and feedback in major depressive disorder. J Affect Disord 118: 69-78.

Steele JD, Kumar P, Ebmeier KP (2007). Blunted response to feedback information in depressive illness. Brain 130: 2367-2374.

Stoy M, Schlagenhauf F, Sterzer P, Bermpohl F, Hägele C, Suchotzki K et al (2012). Hyporeactivity of ventral striatum towards incentive stimuli in unmedicated depressed patients normalizes after treatment with escitalopram. J Psychopharmacol 26: 677-688.

Stringaris A, Cohen P, Pine DS, Leibenluft E (2009). Adult outcomes of youth irritability: a 20 -year prospective community-based study. Am J Psychiatry 166: 1048-1054.

Treadway MT, Zald DH (2011). Reconsidering anhedonia in depression: lessons from translational neuroscience. Neurosci Biobehav Rev 35: 537-555.

Wolf DH (2006). Anhedonia in schizophrenia. Curr Psychiatry Rep 8: $322-328$.

Wolf DH, Satterthwaite TD, Kantrowitz JJ, Katchmar N, Vandekar L, Elliott MA et al (2014). Amotivation in schizophrenia: integrated assessment with behavioral, clinical, and imaging measures. Schizophr Bull 40: 1328-1337.

Wolf DH, Turetsky BI, Loughead J, Elliott MA, Pratiwadi R, Gur RE et al (2008). Auditory oddball fMRI in schizophrenia: 
association of negative symptoms with regional hypoactivation to novel distractors. Brain Imaging Behav 2: 132-145.

Yan CG, Cheung B, Kelly C, Colcombe S, Craddock RC, Di Martino A et al (2013a). A comprehensive assessment of regional variation in the impact of head micromovements on functional connectomics. Neuroimage 76C: 183-201.

Yan CG, Craddock RC, He Y, Milham MP (2013b). Addressing head motion dependencies for small-world topologies in functional connectomics. Front Hum Neurosci 7: 910.
Zink CF, Pagnoni G, Chappelow J, Martin-Skurski M, Berns GS (2006). Human striatal activation reflects degree of stimulus saliency. Neuroimage 29: 977-983.

Zink CF, Pagnoni G, Martin ME, Dhamala M, Berns GS (2003). Human striatal response to salient nonrewarding stimuli. J Neurosci 23: 8092-8097.

Zink CF, Pagnoni G, Martin-Skurski ME, Chappelow JC, Berns GS (2004). Human striatal responses to monetary reward depend on saliency. Neuron 42: 509-517.

Supplementary Information accompanies the paper on the Neuropsychopharmacology website (http://www.nature.com/npp) 